\title{
Internalización de las normas laborales internacionales en el marco del TLCAN: el caso mexicano
}

\author{
Kimberly A. Nolan García*
}

\section{RESUMEN}

Con base en la evidencia de las respuestas de México al Acuerdo de Cooperación Laboral de América del Norte (ACLAN), se examina si el acuerdo paralelo laboral ha impulsado a México a adoptar normas internacionales de derechos laborales y, por tanto, si el "modelo de espiral" puede explicar las mejoras en la protección de tales derechos en México. El artículo sugiere que aun cuando los acuerdos laborales son débiles, las redes de defensa transnacionales pueden llevar a los Estados a aceptar las normas laborales internacionales, lo cual puede resultar en el fortalecimiento de las protecciones laborales dentro de un país.

Palabras clave: ACLAN; redes de defensa transnacionales; modelo de espiral; derechos laborales; México.

\section{ABSTRACT}

Based on the Mexico's responses to the North American Agreement on Labor Cooperation (NAALC), this article evaluates whether the parallel labor agreement has spurred Mexico to adopt international labor rights norms and therefore, if the "spiral model" can explain the improved protection of these rights in Mexico. The author suggests that even when labor agreements are weak, transnational defense networks can lead states to accept international labor norms, which can result in strengthening labor protections inside a country.

Key words: NAALC, transnational defense networks, spiral model, labor rights, Mexico

\footnotetext{
* International Studies Division I Division de Estudios Internacionales (DEI), Centro de Investigacion y Docencia Economicas (CIDE), kimberly.nolan@cide.edu
} 
En The Power of Human Rights: International Norms y Domestic Change (1999), Risse, Ropp y Sikkink estudian el efecto que las normas internacionales de derechos humanos tienen sobre las prácticas y políticas nacionales de un país. Mediante la aplicación de un "modelo de espiral" de cinco etapas de la socialización de las normas a las experiencias de seis países, los autores describen un proceso común por el cual las normas internacionales se transmiten a los niveles subnacionales de gobierno para finalmente ser adoptadas o no por los Estados. En este modelo, los defensores transnacionales, los grupos nacionales y los Estados interactúan para persuadir a éstos de que cambien sus prácticas respecto a los derechos humanos, animándolos a actuar en concordancia con los estándares de comportamiento internacionalmente aceptados.

En la etapa inicial del modelo, Risse y Sikkink establecen que una situación de represión dentro de un país obliga a la débil oposición nacional a mirar fuera del Estado para conseguir aliados internacionales que consecuentemente ejerzan presión externa sobre él para que responda a las demandas de los grupos nacionales (Risse, Ropp y Sikkink, 1999: 22; Keck y Sikkink, 1998: 12). ${ }^{1}$ En esta etapa inicial, la interacción discursiva entre los Estados infractores y los defensores transnacionales empuja al Estado en cuestión a que eventualmente acepte las normas internacionales e institucionalice sus recomendaciones. A lo largo del camino, la argumentación, el discurso y la persuasión marcan las cinco etapas del proceso mediante el cual las normas son internalizadas y generan que el comportamiento del Estado se modifique gradualmente.

Estos autores señalan que el modelo puede ser extendido teoréticamente para describir las normas de socialización dentro de otras áreas temáticas (Risse, Ropp y Sikkink, 1999: 238). El proceso de adopción de normas entre Estados debe ser, por lo tanto, similar para los derechos laborales, ya que existen normas y regímenes similares para su protección; de hecho, los derechos laborales se consideran derechos humanos "positivos" de segunda generación. ${ }^{2}$ Al tomar el modelo de espiral de Risse y coautores para aplicarlo al cumplimiento de los derechos laborales, sigo las prescripciones del modelo para evaluar si México está siendo socializado por la comunidad internacional para que adopte los estándares y prácticas internacionales sobre derechos laborales.

\footnotetext{
${ }^{1}$ En donde el modelo boomerang del libro de Keck y Sikkink (1998) describe la defensa a nombre de los grupos políticos internos de oposición, el modelo en espiral está concebido para ilustrar el patrón de socialización de normas del Estado específicamente en cuanto a derechos humanos.

${ }^{2}$ Debido a la importancia de las prácticas laborales para el comercio y el desarrollo económico, los derechos humanos y los derechos laborales se han desarrollado bajo distintas leyes y precedentes legales. De hecho, se hacen cumplir mediante diferentes procesos y mecanismos con distintas jurisdicciones a niveles regionales, internacionales e internos; sin embargo, existe una superposición. Por ejemplo, el derecho al trabajo es parte de la Carta Económica y Social de las Naciones Unidas, así como la Declaración Universal de los Derechos Humanos.
} 
La evidencia proviene de una evaluación de la experiencia de México con el Acuerdo de Cooperación Laboral para América del Norte (ACLAN), un acuerdo laboral paralelo al Tratado de Libre Comercio (TLCAN). En virtud de éste, los Estados socios deben aplicar y cumplir un conjunto de estándares laborales. En caso de no hacerlo, pueden enfrentarse a los procedimientos formales de resolución de disputas y hasta posibles sanciones comerciales. Las redes transnacionales de defensa utilizaron los mecanismos de denuncias ciudadanas para evidenciar las violaciones a los derechos laborales en México (Graubart, 2009) otorgando, así, un lugar destacado a las recurrentes violaciones del derecho a la libertad de asociación, entre otros derechos laborales, no sólo en el debate público sobre el TLCAN, sino también en la relación bilateral México-Estados Unidos. El modelo de espiral ayuda a interpretar si el mecanismo de denuncia del ACLAN resultó efectivo, o no, para generar cambios en las políticas y prácticas de derechos laborales en México ante la presión transnacional.

Me enfocaré en la experiencia de México con el ACLAN, siguiendo el patrón de Risse, Ropp y Sikkink de aplicar el modelo a los "casos menos probables", en los que el rechazo histórico a la intervención en los asuntos internos hace a México especialmente resistente a las críticas internacionales (Baer y Weintraub, 1994; Domínguez y Fernández de Castro, 2009). Asimismo, han pasado veinte años desde que el Congreso estadunidense aprobó la legislación que permitió la apertura de las negociaciones comerciales y que, a su vez, precipitó la formación de la red de defensa de derechos laborales anti-TLCAN. Gracias a este lapso, es posible determinar cómo el proceso de socialización ha evolucionado con los años, indicando el posible lugar de México en las etapas del modelo de espiral.

El artículo comienza presentando las explicaciones teóricas de la difusión de normas entre Estados. Posteriormente, describo el modelo de espiral de la socialización de normas propuesto por Risse, Ropp y Sikkink, y sugiero cómo podría ser ampliado para explicar los cambios con respecto a la protección de los derechos laborales. Después de presentar la evolución de una red transnacional de defensa en torno a cuestiones no comerciales del TLCAN, analizo cómo las resoluciones de algunos casos ejercieron presión para que México mejorara sus prácticas y políticas sobre derechos laborales. Me ocupo de la violación del derecho de libre asociación por dos razones. Primero porque la libertad de asociación se considera la norma laboral más importante y de la cual derivan los otros derechos fundamentales: si los trabajadores tienen el derecho de formar un sindicato, los otros derechos laborales pueden obtenerse por medio de la organización colectiva. En segundo lugar, la libertad de asociación es el área de los derechos laborales más discutida en el TLCAN, especialmente en el caso de México. Prácticamente todas las denuncias levantadas contra México señalan como principal queja la violación del derecho de libre asociación, sola o como 
parte de un reclamo mayor. Para entender la creciente aceptación de las normas laborales internacionales en México, hay que entender cómo es que México adoptó la norma laboral internacional más importante, que es la que continuamente viola.

Finalmente, aunque el modelo de espiral proporciona un marco para evaluar hasta qué punto los Estados pueden llegar a interiorizar las normas, el argumento presentado aquí sostiene que dicho modelo no aísla con precisión los mecanismos causales por los cuales México comenzó a realizar cambios institucionales en materia de sus prácticas laborales. El artículo concluye que, a pesar de los esfuerzos por mejorar la aplicación de los derechos laborales en México, la evidencia sugiere que el país está todavía en el camino hacia la socialización de las normas laborales.

\section{NORMAS, IDENTIDADES DEL ESTADO Y CAMBIO POLÍTICO}

El estudio de la socialización de normas como explicación del comportamiento estatal se genera a partir de las insuficiencias en las perspectivas neoliberales y neorrealistas para explicar la gama de comportamiento estatal en el sistema internacional (Finnemore, 1996; Kowert y Legro, 1996). Neorrealistas y neoliberales prevén que los Estados se comportan de manera predecible con limitaciones estructurales similares y condiciones sistémicas del sistema internacional. Sin embargo, se observa que los Estados que enfrentan presiones y limitaciones similares responden de manera distinta ante éstas, creando así un enigma para ambas vertientes teóricas (Kowert y Legro, 1996). Los constructivistas sostienen que las ideas desempeñan un papel importante, en lugar de los intereses materiales, en la explicación de este enigma. A pesar de las limitaciones estructurales del sistema internacional que condicionan los intereses de los Estados, éstos pueden crear preferencias endógenas y generan sus propios objetivos e incentivos, con las acciones correspondientes para alcanzar sus metas (Kowert y Legro, 1996).

En el campo de las relaciones internacionales, las normas se describen comúnmente como expectativas compartidas sobre el comportamiento de la identidad de un Estado determinado (Jepperson et al., 1996). Las normas son constitutivas en la medida en que definen y determinan qué identidades de Estado, objetivos y prácticas son legítimos en el sistema internacional (Kowert y Legro, 1996: 453; Jepperson et al., 1996: 54). Las normas también son regulativas en el sentido de que establecen reglas de comportamiento para los Estados, determinando, de esta manera, los roles sociales y acciones aceptadas para aquéllos (Jepperson et al., 1996; Kowert y Legro, 1996). En conjunto, las normas establecen las expectativas sobre quiénes serán los actores legítimos en el sistema internacional, así como también qué tipo de acciones 
llevarán a cabo. Entonces, las normas pueden guiar a los Estados hacia la elección de políticas que encarnen las identidades estatales preferidas por otros Estados (Jepperson et al., 1996: 52). Como tales, las identidades estatales dan pie para reflejar los intereses estatales.

La literatura sugiere que los Estados están muy conscientes de cómo son percibidos por otros Estados, y que ajustarán su comportamiento con el fin de reflejar una imagen más alineada a los valores internacionales (Clark et al., 1998; Finnemore, 1996; Gurowitz, 1999; Hafner-Burton, 2008; Price, 1998; Price y Tannewald, 1996). Si los Estados son sensibles respecto a su imagen internacional, se les puede enseñar a cambiar sus preferencias para estar más en línea con los estándares internacionales (Finnemore, 1996). Lo anterior obliga a los Estados a perseguir objetivos basados no sólo en los intereses estatales, sino también en las percepciones que sus acciones puedan generar entre los demás Estados (Price y Tannenwald, 1996; Price, 1998). Dentro de esta cadena de consecuencias, las normas pueden ser decisivas en el cambio de comportamiento de los Estados al hacerlos conscientes de cómo la comunidad internacional los percibe y, posteriormente, prescribir el comportamiento que les haría tener una imagen más favorable.

La evolución de las preferencias internas y de la identidad del Estado, teóricamente, causan cambios políticos, pero a menudo las redes transnacionales de defensa sirven como promotoras del cambio de conducta y transformación real (Finnemore y Sikkink, 1999). Estas redes se diferencian de otros tipos de redes en que se forman alrededor de "ideas basadas en principios"; ideas que asignan valores normativos del bien y el mal o justicia e injusticia con respecto a las acciones de un Estado (Keck y Sikkink, 1998: 1). Las redes funcionan como mensajeros entre los Estados y el sistema internacional, y entre los actores nacionales e internacionales, transmitiendo información sobre normas y su cumplimiento en los distintos niveles.

Las redes transnacionales de defensa tienen tres propósitos: proporcionan información sobre violaciones a los derechos humanos en el Estado involucrado; legitiman los reclamos de los grupos de oposición dentro del Estado, fortaleciendo de esta manera este tipo de reclamos; y por último, desafían a aquellos Estados que violan normas para que modifiquen su comportamiento (Risse et al., 1998: 5). Los defensores internacionales promueven cambios en el comportamiento, proporcionando información a los Estados sobre opciones de política más aceptables y mediante la politización de los temas que deben ser abordados mediante cambios en el comportamiento estatal (Haas, 1989; Joppke, 1998; Keck y Sikkink, 1998; Price, 1998). Entonces, los defensores alientan algunas identidades estatales, y no otras, mediante la creación de grupos internos y externos; tratando a los Estados que rompen las normas como problemáticos, y tratando a aquellos Estados que aceptan las normas y prácti- 
cas compartidas como miembros integrales de la comunidad internacional (Kowert y Legro, 1996; Price, 1998; Keck y Sikkink, 1998; Hafner Burton, 2008).

El intercambio de información es la base de la red transnacional de defensa. Con un acceso privilegiado a los actores afectados por las violaciones de normas, las redes son capaces de generar información políticamente útil, interpretar los temas en cuestión y, posteriormente, convencer a otros para actuar de acuerdo con los valores compartidos por los miembros de la red. Al mostrar a los Estados cómo perciben las audiencias internacionales su comportamiento, la presión de las redes añade un impulso externo para que los Estados cambien sus políticas y prácticas.

\section{EL MODELO ESPIRAL DE LA SOCIALIZACIÓN DE NORMAS}

El modelo de espiral de la socialización de normas (Risse et al., 1999) ilustra el proceso mediante el cual los defensores transnacionales, los grupos nacionales y los Estados interactúan para persuadir a los gobiernos de actuar conforme a las normas internacionales de derechos humanos. Las normas son internalizadas e institucionalizadas por los Estados a lo largo de cinco etapas.

\section{Primera etapa: la represión}

La primera etapa del modelo se caracteriza por la represión política. Al igual que en el boomerang descrito por Keck y Sikkink (1998), una situación represiva de derechos humanos dentro de un país obliga al grupo de oposición interna débil a buscar fuera del Estado aliados internacionales, incluyendo actores no estatales, organizaciones internacionales y otros Estados. Los canales de comunicación internos suelen estar bloqueados, ya sea porque la oposición interna es demasiado débil para ejercer con éxito su propio programa o porque la naturaleza represiva del contexto les impide hacerlo. Sólo hasta que la red transnacional de defensa logra recopilar información sobre las condiciones dentro del Estado puede, entonces, catalogarlo como infractor de las normas de la comunidad internacional (Risse et al., 1999: 22). Los aliados de la comunidad internacional, a continuación, ejercen presión hacia el Estado en cuestión para que éste modifique su comportamiento represivo (Risse et al., 1999: 22; Keck y Sikkink, 1998: 12). 


\section{Segunda etapa: la negación}

En la segunda etapa, la red transnacional de defensa, una vez formada, recopila información acerca de la situación dentro del país en cuestión para posteriormente dirigirla a foros internacionales. Las redes de defensa convencen a los Estados que cumplen las normas a unirse para presionar al Estado infractor; de esta manera, demuestran a éste que sus acciones están fuera de la esfera de conducta aceptada (Risse et al., 1999: 23). Los Estados observados reaccionan a las demandas realizadas por las redes transnacionales de defensa; primero, negando la validez de las demandas o reclamos y, luego, poniendo en duda la legitimidad de los participantes de la red y los otros Estados al interferir en sus asuntos internos (Risse et al., 1999: 22). Advierten que la negación refleja las etapas iniciales del proceso de socialización porque los Estados no necesitarían justificar sus acciones si fueran impermeables a la socialización o a la crítica de otros Estados (Risse et al., 1999: 23). Que la presión internacional pueda hacer avanzar el proceso de socialización hacia la siguiente fase depende de los tipos de presión que pueda ejercerse en los Estados elegidos y de cuán sensibles sean esos Estados a la presión. Por ejemplo, si se contemplan presiones materiales, como sanciones económicas, los Estados que sufran pérdidas económicas intentarán resolverlas para así responder a las críticas. Del mismo modo, los Estados sensibles a su reputación internacional podrían elegir preservarla y responder a las demandas de actores externos, con lo cual el proceso de socialización pasaría a su tercera fase.

\section{Tercera etapa: concesiones tácticas}

La tercera etapa del modelo predice que el Estado responderá a la presión internacional haciendo "cambios cosméticos" destinados a desviar las críticas, sin dejar de violar las normas en cuestión. En las fases iniciales de la tercera etapa, los Estados pueden responder a la presión de las redes desde una posición instrumental o estratégica, efectuando cambios de comportamiento simplemente para desviar la crítica internacional y no como muestra real de que han aceptado la norma (Risse et al., 1999: 25). Sin embargo, la apertura que se produce cuando los Estados reducen la represión únicamente sirve para otorgar espacio a los grupos nacionales para que se reagrupen. Si el Estado no responde a esta renovada movilización con la represión, la atención internacional da a los grupos nacionales el espacio y la legitimidad para presionar al gobierno "desde abajo", que cuando se combina con la presión simultánea del sistema internacional "desde arriba" (Brysk, 1993), puede causar que los Estados reevalúen sus intransigencias (Risse et al., 1999: 26). 
En esta etapa, los Estados dejan de negar la validez de la norma al hacer cambios cosméticos, y dejan de rechazar que las violaciones tuvieron lugar. En cambio, comienzan a responder a sus críticos, justificando sus actos y señalando los cambios instrumentales y cosméticos de las prácticas para mostrar que la situación de las violaciones a los derechos está mejorando, aun si sólo se trata de cambios cosméticos destinados a descartar críticas posteriores (Risse et al., 1999: 27). Al mismo tiempo, el espacio que se crea al interior y el apoyo a los actores internos que provienen del sistema internacional permiten que los grupos de oposición legitimen a otros actores que deben ser tomados en serio internamente. En las últimas fases de la tercera etapa, las demandas de estos actores comienzan a ser tomadas en cuenta, pues los Estados comienzan a implementar normas en la siguiente fase.

\section{Cuarta etapa: estatus prescriptivo}

De acuerdo con el modelo, los Estados hacen la transición hacia la institucionalización de las normas en la cuarta etapa. En este punto del proceso de socialización, los Estados modifican su comportamiento, en menor medida, a partir de un posicionamiento estratégico y empiezan un proceso en el que asumen la adopción de normas y su cumplimiento (Risse et al., 1999). El proceso discursivo de responder a las redes transnacionales, organizaciones internacionales y otros Estados implica que los gobiernos ya no pueden ignorar las críticas y el intercambio en el debate. En esta etapa, los Estados regularmente se refieren a las normas para describir su comportamiento como propio de la tercera etapa; la validez de la norma no es controversial, como lo era en la segunda. Sin embargo, es importante observar que en las primeras fases de esta etapa, los Estados dan a las normas carácter legal, incluso cuando continúan violándolas.

Los autores determinan que las acciones de los Estados en este punto indican cuándo han entrado en esta etapa, incluyendo la ratificación de convenciones internacionales, la creación de nuevas leyes federales o la implementación de nuevos mecanismos para el cumplimiento interno (Risse et al., 1999). ${ }^{3}$

En esta etapa no es tan importante que los Estados "crean" en las normas que ahora apoyan (Risse et al., 1999: 29); nunca pueden estar plenamente convencidos de aceptar estas normas (Hafner-Burton, 2005). Lo más importante es que las palabras y los actos concuerden, es decir, los Estados hablan del cumplimiento de normas mientras intentan cumplir con las obligaciones internacionales y regionales, y comienzan a implementar normas internamente (Risse et al., 1999: 30). Sin embargo, es importante

\footnotetext{
${ }^{3}$ Agradezco al dictaminador por recordarme esta distinción crucial entre las etapas cuatro y cinco.
} 
mencionar aquí que incluso cuando firman acuerdos internacionales, dan a las normas la fuerza del derecho internacional o acceden a implementarlas internamente, o están obligados a defenderlas, no significa que en los Estados las prácticas se modifiquen inmediatamente. ${ }^{4}$ La adopción de normas toma tiempo y los Estados pueden permanecer estancados en la cuarta etapa por un periodo largo. Parte del problema es que la adopción de las normas lleva tiempos distintos entre las dependencias y agencias (Shor, 2008; Aspinwall, 2013), un aspecto de socialización de las normas contemplado por los autores, pero que no está incluido en el modelo. ${ }^{5}$

El modelo de espiral sugiere que cuando la presión externa los obliga a cambiar su comportamiento, los Estados se mueven de manera uniforme hacia la adopción de las normas, pasando por las etapas iniciales hasta llegar a la quinta etapa, en la cual aceptan la validez de la norma y trabajan para implementarla (Risse et al., 1999). Resulta, entonces, difícil reconocer en la práctica en qué etapa del proceso se encuentran o si están avanzando hacia la quinta etapa, por medio de cuáles normas y cómo, debido a las desigualdades del proceso al interior de los Estados.

En consecuencia, ¿cómo podríamos saber que los Estados están entrando en una fase de adopción de las normas laborales? Para el caso de estos derechos esta etapa es difícil de identificar, pues los Estados pueden haber firmado previamente las principales convenciones internacionales que señalan su compromiso con los estándares laborales. Sin embargo, para saber si estos compromisos son sinceros, los Estados necesitarían demostrar su cumplimiento interno, ya sea mediante el desarrollo de nuevas prácticas y procedimientos o con la implementación de nuevos programas para abordar cuestiones específicas como el trabajo infantil, por ejemplo. Podríamos reconocer que México esté en transición de la cuarta fase a la siguiente, la aplicación consistente de las normas laborales, cuando las promesas de vigilar el cumplimiento de las normas laborales se vean seguidas de los cambios de las políticas y prácticas iguales a la ley internacional que cumple el país y de las nuevas obligaciones en materia de derechos laborales.

\section{Quinta etapa: comportamiento consistente con la norma}

Las presiones desde abajo y desde arriba continúan, permitiendo la implementación de nuevas prácticas que sustentan la norma. Los autores describen esta etapa final del

\footnotetext{
${ }^{4}$ Los trabajos recientes sobre el respeto a los derechos humanos observan que la ratificación de convenciones internacionales sobre derechos humanos muchas veces lleva al incremento de las violaciones de éstos que lo que sucedía antes, ya que los Estados firman acuerdos internacionales sin tener intenciones de cumplir sus regulaciones (Hill, 2009; Hathaway y Oona, 2002; Camp Keith, 1999).

${ }^{5}$ Risse et al. (1999: 31) atribuyen una adopción desigual de las normas para los principales problemas de comando de agentes en el control militar civil.
} 
modelo como el punto en el cual los Estados aceptan la validez de la norma y trabajan para implementarla en todos los niveles (Risse et al., 1999). El cumplimiento de la norma se convierte, entonces, en una práctica habitual: el comportamiento del Estado refleja la norma. Finalmente, ésta se respalda por el Estado de derecho, lo que sugiere que la institucionalización es casi completa. En el área de derechos laborales, puede observarse el cumplimiento de las normas mediante el monitoreo de los discursos de los funcionarios de gobierno para ver si hacen referencia a la norma, y también evaluando si las prácticas de derechos laborales han mejorado a lo largo del tiempo.

\section{REDES TRANSNACIONALES DE DEFENSA}

\section{EN LA NEGOCIACIÓN DEL TLCAN}

Cuando el representante de Comercio de Estados Unidos (United States Trade Representative, USTR) reveló que ese país pretendía buscar un acuerdo comercial con México y Canadá, las organizaciones sindicales de Estados Unidos reaccionaron ante el anuncio de manera inmediata y negativa. La ampliación del acuerdo comercial, ya existente con Canadá, para incluir a México, podría empujar algunos empleos de Estados Unidos hacia México, tal como lo había hecho el Programa de Industrialización Fronteriza de 1965. Las organizaciones sindicales estadunidenses participaron en la creación de una red de organizaciones que trabajaron para cambiar el debate sobre el TLCAN y para hacer fracasar el acuerdo. Ambientalistas, grupos de derechos humanos, comunidades religiosas y organizaciones comunitarias también se unieron a la red formada en la primavera de 1991, haciéndose llamar Mobilization on Development, Trade, Labor and the Environment (MODTLE, por sus siglas en inglés) (Mayer, 1998: 70). Participantes de las principales redes, incluyendo International Labor Education and Research Fund (ILRF), Greenpeace, United Auto Workers, AFL-CIO, Natural Resources Defense Council, Family Farm Coalition, entre otros, convocaron a un foro sobre el impacto del TLCAN en el trabajo, la agricultura y el medio ambiente (Mayer, 1998: 76). Decidieron que primero se concentrarían en bloquear los esfuerzos del presidente Bush para solicitar la autorización del Congreso respecto a la negociación del acuerdo.

En Canadá, la oposición al libre comercio ya se había atrincherado. Veían al TLCAN como una extensión del TLC entre Canadá y Estados Unidos, al cual se habían opuesto contundentemente. Los sindicatos canadienses consideraron que unos lazos más estrechos con Estados Unidos acelerarían la competencia y que se perderían empleos canadienses ante los trabajadores estadunidenses. El Congreso Laboral Canadiense insistió en que deberían desarrollar una oposición al TLC desde todos los frentes 
(Robinson, 2002: 123). Sin embargo, la Confederación Sindical Nacional de Quebec (CSN) mantuvo una posición más moderada, como la de los sindicatos en Estados Unidos, y esperó influenciar los términos del acuerdo (Robinson, 2002: 124). Al mismo tiempo, Pro-Canada Network, una organización no gubernamental (onG) que lideraba la lucha en contra del TLC, resurgió como Action Canada Network, y comenzó a levantar oposición total también al TLCAN en Canadá.

La Confederación de Trabajadores de México (Стм) apoyó los esfuerzos del partido en el poder para promover el acuerdo comercial, por lo que el Frente Auténtico del Trabajo (FAT) se encargó de representar a la oposición sindical de rank and file. La Red Mexicana de Acción Frente al Libre Comercio (RMALC), una entidad que agrupaba casi cien organizaciones de trabajadores y campesinos independientes, indígenas, grupos ambientales y asociaciones civiles, surgió para encabezar la oposición en nombre de la sociedad civil. Posteriormente, realizó foros públicos sobre los impactos negativos e inminentes del TLCAN en México (RMALC, 2003).

Una vez que estos grupos formularon estrategias nacionales, encontraron maneras de trabajar juntos para que, en 1992, la red inicial de activistas en cada Estado asumiera formas de organización transnacional. Las ONG de los tres países establecieron los contactos diarios necesarios para desarrollar un plan de acción común y fijar objetivos para influir en el TLCAN (Cook, 1997: 519). Estos grupos fueron cruciales para suministrar información a los ciudadanos y a los legisladores sobre el contenido del acuerdo, así como los posibles impactos del libre comercio sobre la economía, la sociedad y la cultura de cada una de las naciones involucradas. Algunos grupos en México comenzaron a acercarse a los inmigrantes mexicanos que radicaban en Estados Unidos, mientras otros cabildeaban en el Congreso de ese país sobre los impactos del TLCAN en México, incluso organizando visitas para los legisladores (Cook, 1997). Así, una oleada de oposición al acuerdo comercial se había movilizado en las tres naciones.

Mientras que las redes transnacionales se movilizaron en contra del TLCAN, el debate en Estados Unidos en general se dio en torno a la falta de respeto al Estado de derecho y corrupción en México, con el fin de cuestionar si este país debería ser recompensado por su pobre desempeño democrático con un acuerdo comercial (Mayer, 1998; Baer y Weintraub, 1994). De acuerdo con el modelo en espiral, los Estados reaccionan a la presión transnacional primero negando los cargos y apelando a la no injerencia internacional en sus asuntos nacionales. Pero, en este caso, el gobierno de Salinas no negó que la aplicación de la legislación mexicana o sus instituciones democráticas necesitarían mejorar; al contrario, el gobierno fue sensible a las críticas hechas por aquellos grupos. Si bien hasta ese momento la participación de México en foros internacionales y sus relaciones individuales con determinados Estados se habían visto limitadas por los principios de no intervención y de autodeterminación de los Esta- 
dos en materia de política exterior, hacia el final de la década de 1980 esto comenzó a cambiar. Salinas tenía la intención de modificar el carácter de las relaciones entre México y Estados Unidos, que durante esa década habían sido conflictivas, de tal modo que el acuerdo comercial serviría para solidificar una relación positiva con la potencia (Domínguez y Fernández de Castro, 2009). Así que el gobierno no negó que hubiera problemas, ni cuestionó la legitimidad de esos grupos por criticar la política interna de México; más bien, empezó a implementar algunas reformas para acallar esas críticas.

Salinas respondió a las inquietudes de Estados Unidos respecto a la debilidad de las leyes ambientales mexicanas mediante una revisión de la legislación ambiental y tomando "medidas muy visibles" para responder a las violaciones recurrentes (Cook, 1997: 521). Respecto a las preocupaciones por el tráfico de drogas, corrupción y violaciones a los derechos humanos, se hizo una purga de la policía judicial, y aumentaron los esfuerzos de interdicción contra los traficantes de drogas (Cook, 1997: 521). Para demostrar a ese país que México estaba dispuesto a trabajar en materia de derechos humanos (una de las mayores preocupaciones de Estados Unidos), en 1990 se creó la Comisión Nacional de Derechos Humanos (CNDH), autónoma con respecto a la Secretaría de Gobernación (Covarrubias Velasco, 1999), y la Procuraduría General de la República nombró a un defensor de los derechos humanos (Cook, 1997).

En el contexto de la negociación del TLCAN, México utilizó estas reformas para mostrar su voluntad de resolver algunos problemas para cambiar su imagen frente a Estados Unidos antes de entrar a las negociaciones del tratado, pero nunca intentó reformar a gran escala la corrupción política que permitió el surgimiento de algunos problemas; por eso, deben ser consideradas reformas superficiales, no profundas.

Es interesante observar que estas reformas no alcanzaban las cuestiones de derechos laborales. La principal negativa al TLCAN en Estados Unidos se basaba en la oposición sindical a causa de la precaria situación en materia de derechos laborales en México (y, en segundo término, por la falta de protección ambiental), pero la administración de este país nunca respondió a estas críticas con algún plan para mejorar las garantías o las prácticas laborales. De hecho, cuando comenzaron las negociaciones sobre materia laboral, casi abandonó la mesa de negociaciones al objetar la propuesta de mayor escrutinio por parte de Estados Unidos y Canadá en las relaciones industriales de México (Cameron y Tomlin, 2000).

La red, en algunos casos, influyó en los miembros del Congreso estadunidense para condicionar la autoridad de negociación al presidente (autoridad fast track) con respecto a la inclusión de una cláusula social. Los senadores del Partido Demócrata pidieron acuerdos paralelos sobre el trabajo y el medio ambiente que incluyeran un mecanismo de resolución de controversias, con el poder de castigar con sanciones las violaciones a las nuevas normas, y así armonizar las protecciones en las tres naciones 
(Lowe, 1997; Hafner-Burton, 2009). A fin de obtener los votos necesarios para aprobar el fast track, la administración de Bush aceptó este plan de acción. Mientras las negociaciones del TLCAN llegaban a su fin, comenzaban las reuniones sobre el acuerdo laboral y la cláusula de cooperación ambiental (Cameron y Tomlin, 2000). La red transnacional determinó que el nuevo proceso para la resolución de conflictos, incluido en los dos acuerdos paralelos, ofrecía un nuevo enfoque sobre dónde construir las relaciones que se habían forjado en las campañas anti-TLCAN. ${ }^{6}$ En lugar de disipar la oposición, la aprobación del tratado fortaleció a los grupos de derechos laborales, ya que el nuevo mecanismo para la resolución de conflictos proveyó una oportunidad política para que el acuerdo paralelo hiciera cumplir adecuadamente los principios de derechos laborales incluidos en él (Kay, 2005, 2011; Stillerman, 2003). Las redes comenzaron a colaborar para construir y presentar los casos que pondrían a prueba la voluntad de los paneles del TLCAN en la promoción y protección de los derechos laborales en América del Norte.

\section{MÉXICO ANTE EL ACLAN}

A pesar de que las confederaciones laborales fueron invitadas a participar en el proceso de negociación, el ACLAN fue mucho menos completo de como las coaliciones hubieran deseado. ${ }^{7}$ En lugar de desarrollar nuevas normas laborales regionales, el acuerdo laboral paralelo únicamente obligó a las tres naciones a adoptar una "aplicación efectiva" de sus propias leyes laborales nacionales (Compa, 2001).

Aunque los derechos laborales fundamentales están escritos en la Ley Federal del Trabajo, y México ha firmado la mayoría de los convenios laborales fundamentales de la Organización Internacional del Trabajo (отт) sobre derechos laborales, su aplicación es perennemente débil. El proceso del ACLAN proporcionó una potencial segunda fase de compromiso sobre derechos laborales en los tres países. Bajo el proceso del arbitraje del acuerdo laboral, cualquier ciudadano o grupo puede presentar una queja ante la Oficina Administrativa Nacional (NAO), oficinas que el TLC estableció en cada estado para administrar los procesos de arbitraje. ${ }^{8}$ Al recibir una queja,

\footnotetext{
${ }^{6}$ Hay una gran cantidad de estudios de caso en que se argumenta que la experiencia con el TLCAN sirvió como catalizador para la creación o renovación de los vínculos sindicales transfronterizos; véase Babson (2002); Compa (2001); Cook (1997); Hathaway (2002); Juárez Núñez (2002); Kay (2005, 2011); Stillerman (2003); Williams $(1999,2003)$.

${ }^{7}$ Debido a que la стм apoyó la postura del gobierno sobre el TLCAN y México no se fiaba de los acuerdos paralelos en general, no participó en la discusión sobre las cláusulas laborales.

${ }^{8}$ Graubart (2008) proporciona un análisis completo sobre las instituciones que participan de los acuerdos laborales y ambientales y sus mecanismos de aplicación. La NAO en Estados Unidos está ahora a cargo de la aplicación de otras cláusulas laborales y se llama Office of Trade Agreement Implementation (OTAI).
} 
una NAO primero determina si se debe revisar con mayor profundidad la denuncia. Si la NAO estadunidense o canadiense decide revisar el caso, se convoca a una audiencia pública con las partes interesadas e involucradas en la queja y se analizan las alegaciones contenidas en la queja. ${ }^{9}$ Después, se corroboran las afirmaciones para determinar si las acciones del Estado, en el caso presentado, fueron consistentes con las leyes laborales nacionales. ${ }^{10}$ Por último, la NAO hace público un informe sobre cómo abordar las cuestiones planteadas en el caso.

Las oficinas de la NAO pueden sugerir tres formas de abordar los temas incluidos en las quejas con los países que han cometido violaciones. Para violaciones de salud y seguridad laboral se encuentra disponible toda una gama de remedios que incluyen: las consultas ministeriales, la revisión del caso por un panel de expertos en asuntos laborales, el caso puede ser sometido a un proceso formal de solución de controversias, o la aplicación de multas y sanciones comerciales. ${ }^{11}$ Los asuntos que involucran trabajo infantil, disputas sobre el salario mínimo o violaciones de seguridad y salud están sujetos a la solución de controversias, y si aún continúan sin ser resueltas, pueden entran en vigor las multas y sanciones comerciales (North American Agreement on Labor Cooperation -NAALC-, 1993). Los asuntos relativos a "técnicas laborales", tales como trabajo forzado, condiciones mínimas de trabajo, discriminación, remuneración de los trabajadores o protección de migrantes se limitan a las consultas ministeriales y evaluación por el panel de expertos (NAALC, 1993). Los derechos colectivos del trabajo, incluyendo la libertad de asociación, el derecho a organizarse y la negociación colectiva, gozan de la menor compensación, pues sólo pueden acogerse a las consultas ministeriales. ${ }^{12}$

Las redes transnacionales utilizaron el proceso de arbitraje como un área potencial para ser aplicada y para presionar a México en el cumplimiento de las normas laborales. Hasta 2012, se habían presentado cuarenta y un peticiones al ACLAN, en su mayoría en contra de México, y principalmente ante la NAO de Estados Unidos. ${ }^{13}$ La mayoría de los casos han sido entregados por las mismas ONG, defensores de derechos laborales, grupos de derechos humanos y sindicatos que encabezaron el movimiento para detener el tratado comercial. El cuadro 1 presenta información descriptiva

\footnotetext{
${ }^{9}$ Los casos en contra de México sólo pueden ser revisados por las NAO de Canadá o Estados Unidos.

${ }^{10}$ La NAO de México no convoca ni participa en las audiencias públicas, ya que las considera fuera de los requisitos de procedimiento del acuerdo (NAO México, 2006).

${ }^{11}$ Las consultas ministeriales son reuniones entre los ministros de Trabajo de los Estados involucrados, y los resultados de dichas consultas son los pronunciamientos oficiales sobre cómo podría resolverse el caso. Bajo ciertas circunstancias, los ministros pueden llegar a tener una sesión especial de consejo ministerial para medidas más rigurosas (NAALC, 1993).

${ }^{12}$ Los críticos vieron el acuerdo como una forma de diluir la legislación nacional mediante el desarrollo de tres tipos de resoluciones para diferentes violaciones de derechos laborales.

${ }^{13}$ En Nolan Garcia (2011) se encuentra un apéndice con un recuento de los casos presentados hasta 2010.
} 
sobre los casos que fueron aceptados de los cuarenta y un sometidos al proceso de revisión por los comités trinacionales de arbitraje laboral.

\begin{tabular}{|c|c|c|c|}
\hline \multicolumn{4}{|c|}{$\begin{array}{c}\text { Cuadro } 1 \\
\text { CASOS SOMETIDOS AL PROCESO DEL ACLAN, 1994-2012 }\end{array}$} \\
\hline Año & Nombre del caso & Estado & Violaciones laborales \\
\hline 1994 & $\begin{array}{l}\text { Honeywell } \\
\text { General Electric } \\
\text { Sony }\end{array}$ & México & $\begin{array}{l}\text { Derecho a la libre asociación, estándares mínimos } \\
\text { de empleo }\end{array}$ \\
\hline 1995 & Sprint & EU & Derecho a la libre asociación \\
\hline 1996 & $\begin{array}{l}\text { SUTSP } \\
\text { Maxi-Switch }\end{array}$ & México & $\begin{array}{l}\text { Derecho a la libre asociación } \\
\text { Imparcialidad de la junta local de conciliación y } \\
\text { arbitraje (JLCA) }\end{array}$ \\
\hline \multirow[t]{2}{*}{1997} & $\begin{array}{l}\text { Gender } \\
\text { ITAPSA* }\end{array}$ & \multirow[t]{2}{*}{ México } & $\begin{array}{l}\text { Discriminación } \\
\text { Derecho a la libre asociación, salud y seguridad } \\
\text { ocupacional }\end{array}$ \\
\hline & Han Young ** & & $\begin{array}{l}\text { Derecho a la libre asociación, salud y seguridad } \\
\text { ocupacional, imparcialidad de la JLCA }\end{array}$ \\
\hline \multirow{4}{*}{1998} & SOLEC & \multirow{4}{*}{ EU } & $\begin{array}{l}\text { Derecho a la libre asociación, estándares mínimos } \\
\text { de empleo, salud y seguridad ocupacional, discri- } \\
\text { minación }\end{array}$ \\
\hline & Apple Growers & & \multirow{3}{*}{ Derecho del migrante } \\
\hline & De Coster Egg & & \\
\hline & Yale ıns* & & \\
\hline 1999 & TAESA & México & $\begin{array}{l}\text { Derecho a la libre asociación, estándares mínimos } \\
\text { de empleo, salud y seguridad ocupacional, impar- } \\
\text { cialidad de la JLCA }\end{array}$ \\
\hline 2000 & Auto Trim & México & Salud y seguridad ocupacional \\
\hline 2001 & New York State & EU & Compensación de trabajadores \\
\hline 2003 & Puebla, *** & México & $\begin{array}{l}\text { Derecho a la libre asociación, estándares mínimos } \\
\text { de empleo, salud y seguridad ocupacional, impar- } \\
\text { cialidad de la JLCA }\end{array}$ \\
\hline 2003 & North Carolina & EU & Derecho del migrante \\
\hline \multirow[t]{2}{*}{2005} & Hidalgo & México & $\begin{array}{l}\text { Derecho a la libre asociación, estándares mínimos } \\
\text { de empleo, salud y seguridad ocupacional, impar- } \\
\text { cialidad de la JLCA, discriminación, trabajo infantil }\end{array}$ \\
\hline & H2B Visa Workers & EU & Derecho del migrante, trabajo forzado \\
\hline
\end{tabular}




\begin{tabular}{|llll|}
\hline & CASOS SOMETIDOS AL PROCESO DEL ACLAN, 1994-2012 & $\begin{array}{c}\text { Cuadro 1 } \\
\text { (continuación) }\end{array}$ \\
Año & Nombre del caso & Estado & Violaciones laborales \\
2006 & $\begin{array}{l}\text { North Carolina } \\
\text { Public Employees }\end{array}$ & EU & Derecho a la negociación colectiva \\
2008 & North Carolina & EU & Derecho a la negociación colectiva \\
2010 & SME* & México & Derecho a la libre asociación \\
2011 & H2B visas & EU & Derecho del migrante \\
2012 & Alabama H5.6 & EU & Derecho del migrante \\
\hline * Denota un caso sometido a una revisión en la nAo de Estados Unidos y la de Canadá. \\
** Denota que está compuesto de dos entregas, el caso original y un suplemento de alegaciones adicionales.
\end{tabular}

De todas las peticiones entregadas, veinticinco, esto es, el 61 por ciento, fueron aceptadas para una revisión formal. ${ }^{14}$ Como se ilustra en el cuadro, la libertad de asociación surgió como la principal queja registrada en la mayor parte de los casos aceptados por las NAO (doce en total), pero especialmente entre los presentados en contra de México. La mayoría de querellas, en torno a temas de libertad de asociación, refleja la rivalidad política entre sindicatos independientes y sindicatos asociados con el sistema corporativista de este país. La violación del derecho de asociación en México se debe con frecuencia a las disputas sobre los procedimientos de registro de sindicatos independientes que pertenecen al sistema corporativista. La pérdida de recursos económicos para apoyar el clientelismo político tras las crisis económicas de finales de la década de los años ochenta y principios de los noventa ha contribuido a disminuir la dominación política del Partido Revolucionario Institucional (PRI) a nivel nacional (Shirk y Edmonds-Poli, 2009). Con menos recursos, el partido es cada vez menos capaz de proporcionar beneficios materiales y subsidios gubernamentales a sus clientes, incluyendo a los sindicatos corporativistas. ${ }^{15}$

Si bien el sistema de partidos en México ha cambiado radicalmente desde el año 2000 hasta convertirse en un verdadero sistema de varios partidos, en donde el PRI no se considera más una fuerza política hegemónica, al menos a nivel nacional, las fuerzas democratizantes que alteraron el sistema de partidos no han participado en

\footnotetext{
14 Tres de los casos: ITAPSA, de 1997; INS, de 1998, y el caso de Puebla, en 2003, se presentaron simultáneamente en dos oficinas de la NAO para dos revisiones independientes.

${ }^{15}$ Mientras que la стм todavía era capaz de mantener la representación política (Murillo, 2001), la disminución del peso electoral del PRI en el tiempo significó que su canal de poder estaba en peligro. En las décadas de 1970 y 1980, la cTM tenía alrededor de noventa de los escaños adjudicados al PRI; en el 2000, no tuvo más de diecinueve (Shirk y Edmonds-Poli, 2009).
} 
la política laboral de alguna manera significativa (Roman y Velasco Arregui, 2006). Los sindicatos de la стм no están dispuestos todavía a permitir que los sindicatos independientes ganen terreno en áreas donde mantienen el control monolítico de contratos, en parte para mantener lo que queda de la base política del poder local del partido, y en parte, también, para mantener el peso del partido en el gobierno.

La mayor herramienta de la que dispone el Estado para limitar la sindicalización independiente es el control sobre la administración de las juntas de conciliación y arbitraje (JCA) que regulan el sistema de relaciones laborales. Como es una estructura tripartita, el representante de los trabajadores casi siempre se elige de entre las filas de los más influyentes de la confederación sindical, lo que, por lo general, tiene como resultado una representación sindical tomada de las filas corporativistas. Los trabajadores que se organizan fuera del sistema corporativista a menudo son frustrados por agentes estatales de las juntas locales de conciliación y arbitraje (JLCA), que en ocasiones conspiraron con la administración para negar el registro de sindicatos independientes como entidades legales.

Para los casos en que conciernen cuestiones de libertad sindical, el único recurso posible son las consultas ministeriales, juntas formales entre los secretarios de Trabajo para discutir los problemas asociados con el derecho de libre asociación en América del Norte. Como parte de algunas resoluciones de casos que terminaron en consultas ministeriales, los gobiernos de Estados Unidos y México, y en ocasiones Canadá, han acordado planes de acción detallados para discutir aquellas cuestiones laborales que se ponen de manifiesto en los asuntos que llegan a través del proceso del ACLAN. Desde 1995, los gobiernos de México y Estados Unidos han firmado varios acuerdos que reiteran su compromiso con la libertad de asociación, primero como parte de la resolución del caso Sony, y de nuevo en 1996 para el caso Sprint, en 1997 para el caso SUTSP, y en 2000 para los casos de ITAPSA y Han Young (Nolan Garcia, 2011). ${ }^{16}$

El caso de la empresa ITAPSA destaca no solamente por demostrar el problema de la colusión de la JLCA para exacerbar las rivalidades sindicales y las dificultades de registrar un sindicato independiente en México, sino porque representa, con mucho, el mayor número de patrocinadores que cualquier otro caso. Sesenta y cinco grupos en Estados Unidos, México y Canadá firmaron una petición sobre la violación del derecho de libre asociación en ITAPSA, y fue presentado tanto en la NAO de Estados Unidos como en la de Canadá en el año 1997. Aunque el asunto terminó formalmente en consultas ministeriales (como marca el límite para los casos de libertad de asociación), como

\footnotetext{
${ }^{16}$ Hasta la fecha, los ministros de Trabajo han firmado siete acuerdos ministeriales que los comprometen a implementar actividades de cooperación para educar al público, o entre sí, sobre los aspectos técnicos de la regulación de los derechos laborales y su aplicación en cada Estado.
} 
parte de la resolución México firmó un acuerdo ministerial en el cual se comprometió a promover el registro público de los contratos colectivos. A cambio, la Secretaría del Trabajo y Previsión Social (STPS) desarrolló dos sitios web para el registro de contratos: uno permite acceso público a los documentos de registro de los sindicatos, y el otro es una base de datos de los contratos colectivos con información sobre quién posee los derechos de negociación dentro de las fábricas individuales y, en algunos casos, hay acceso a copias de los documentos de registro y los estatutos de cada organización.

El resultado, después de haber recibido y resuelto muchos casos sobre este tema en su contra, serían mejoras en el derecho de libre asociación en México. Más allá de establecer el registro público de sindicatos mencionado arriba, hubo avances en el uso del voto secreto en elecciones sindicales. Muchos casos, incluyendo Maxi-Switch (1996), Han Young (1997), y TAESA (1999), revelaron que durante la elección de la toma de nota, el uso de votos por voz introdujo ciertos elementos de intimidación que luego se convirtió en una de las razones principales de por qué los sindicatos independientes no podían ganar estas elecciones. Citando el Acuerdo Ministerial de 1997 y la declarada intención de México para promover el voto secreto, abogados laborales fueron capaces de lograr un compromiso por parte del gobierno para incluir la libertad de asociación en cualquier agenda política en torno a la reforma laboral (Graubart, 2008). Mientras que todavía no se tocaba el tema de una reforma laboral nacional, la Junta Federal del Trabajo en la Ciudad de México comenzó a ejercer el voto secreto de manera consistente después de 2002 (Hathaway, 2002; Aspinwall, 2013; Maquila Solidarity Network, 2002). Finalmente, la Suprema Corte de Justicia decretó en 2008 que todas las elecciones sindicales deben realizarse por votación secreta, y este procedimiento electoral es ahora una práctica estándar (Aspinwall, 2013).

Una vez que el proceso del ACLAN hizo evidente el trato desigual de los sindicatos independientes frente los funcionarios de las JLCA, el gobierno federal mexicano se sensibilizó más ante la presión emanada del proceso de arbitraje, e inició el camino para democratizar las políticas de regulación sindical. Algunas veces, las autoridades mexicanas fueron más allá de lo estipulado por el ACLAN para responder a las violaciones. Los funcionarios exigían nuevas elecciones sindicales, o reconocían las victorias de la oposición incluso antes de que concluyeran las audiencias de la NAO y se dictaran los pronunciamientos (Compa, 2001).

El caso de Maxi-Switch es un ejemplo. A los trabajadores que intentaron formar un sindicato independiente se les negó el registro en la junta local de Sonora. Cuando el caso llegó al ACLAN y se solicitó una audiencia pública, el gobierno mexicano intervino para resolver la queja, y otorgó el registro al sindicato independiente para evitar la celebración de la audiencia pública (U.S. Department of Labor, 1997). El 16 de abril, dos días antes de que la audiencia pública se llevara a cabo en Washington, la NAO de 
Estados Unidos aprobó la solicitud de retiro de la petición, pues el conflicto laboral había "concluido favorablemente" con el reconocimiento del sindicato independiente (Borderlines, 1997).

El caso Han Young nos provee de otro ejemplo. Después de que la junta local de Tijuana se rehusara a certificar los resultados de una elección en la cual un sindicato independiente había ganado los suficientes votos para la toma de nota, la Fiscalía General de la Nación intervino al emitir acciones de amparo para suspender la decisión de la junta local de Tijuana (Williams, 2003). Esto hizo que el sindicato independiente y sus partidarios presentaran el caso al ACLAN bajo el rubro de libertad de asociación, y fue aceptado para su revisión a finales de 1997. En ese momento, el gobierno federal presionó a la junta local para iniciar una negociación con el sindicato independiente. La dirección de Han Young y los representantes del sindicato rival de la Confederación Revolucionaria de Obreros y Campesinos (CROC) mediaron un acuerdo, el cual incluía una revocación del caso ACLAN a cambio de reconocimiento oficial del sindicato disidente y pagos extrajudiciales a los líderes de la CROC (Williams, 2003).

Estos tres casos son extraordinarios en cuanto a que tuvieron resoluciones que no son representativas de los casos del ACLAN en su conjunto -las cuales a menudo terminan en consultas ministeriales que no alcanzan a incluir cambios de políticas o prácticas laborales que mejoren las condiciones de trabajo en el nivel local, mucho menos en el nacional. Sin embargo, estas resoluciones son importantes ya que muestran que las autoridades mexicanas no sólo fueron sensibles ante las resoluciones dictadas por el proceso del ACLAN, sino que también implementaron acciones fuera de los canales oficiales para resolver los casos. Esto es especialmente interesante porque bajo el acuerdo paralelo, los casos de libertad de asociación tienen el menor número de canales para su resolución, ya que están limitados a las consultas ministeriales; sin embargo, son estos casos los que a menudo tuvieron resoluciones más drásticas fuera de la estructura institucional del ACLAN.

Una vez que los defensores ganaron una apertura en el diálogo con México, fueron entonces capaces de utilizar el proceso del ACLAN para obligar al gobierno mexicano a explicar la incapacidad del sistema de las juntas de conciliación para emitir decisiones imparciales; asimismo, para hacerlo responder por su aquiescencia a las violaciones del derecho a la libertad de asociación, empujando efectivamente al gobierno a explicarse las cuestiones relacionadas con derechos laborales (Graubart, 2008). Considerando que, en el pasado, el debate sobre la reforma laboral siempre había sido una cuestión interna entre las élites políticas de la STPS, la Стм y Coparmex (Zapata, 2006), es importante señalar que ahora, como consecuencia de los referidos avances, la STPS invita a la Unión Nacional de Trabajadores (UNT) para participar en las discusiones formales de las políticas laborales (NAO México, 2006). 
A lo largo del tiempo, y a medida que estas dinámicas políticas iban siendo extendidas al interior del país, los defensores laborales fueron capaces de contribuir al diálogo político y de cabildear para lograr otros cambios importantes, incluso después de que había terminado el proceso de revisión del ACLAN. Un ejemplo de ello es que en 1998, mientras que en el ACLAN revisaban un caso sobre el uso de pruebas de embarazo como parte de la contratación de trabajadoras en el sector de las maquiladoras, se empezó a cambiar esta práctica en México. ${ }^{17}$ Una vez que Estados Unidos y México empezaron las negociaciones bilaterales sobre el tema en el marco de las consultas ministeriales, el gobierno de México hizo público su compromiso de eliminar la discriminación de género en consonancia con los acuerdos internacionales que ha firmado al respecto. Después, creó “Oficinas para asuntos especiales" bajo la sTPs para investigar casos de trabajo infantil, de mujeres en la fuerza laboral y necesidades de los trabajadores con discapacidad (U.S. Department of State, 1999); también se creó una oficina independiente para tratar cuestiones de igualdad de género (U.S. Department of State, 2000).

El caso dio respaldo a los esfuerzos de los grupos feministas en la Ciudad de México para presionar al gobierno y que prohibiera la práctica (Hertel, 2006). En 2002, el gobierno de México firmó un acuerdo con el cual el Consejo Nacional de la Industria Maquiladora comprometió a sus integrantes a poner fin a esa práctica; igualmente, varias empresas de Estados Unidos se comprometieron públicamente a poner fin a las pruebas de embarazo en México. En 2003, la sTPs había firmado trece acuerdos con los gobiernos estatales para comprometerse en el tema (U.S. Department of Labor, 2007). Finalmente, también en 2003, de la Ley Federal para Prevenir y Eliminar la Discriminación entró en vigor, ley que incluye protección contra la prueba de embarazo obligatoria en todo México, de manera que se prohíbe la práctica por primera vez formalmente (U.S. Department of Labor, 2007).

\section{Pasos hacia el respeto de los derechos laborales en México}

Una forma de medir el proceso de socialización de normas es identificar si las acciones que señalan la adhesión a esas normas en el ámbito internacional cumple con las mejoras dentro del país en cuestión. Si México está interiorizando las normas sobre derechos laborales, los efectos se deberían percibir en los intentos por abordar cuestiones sobre su cumplimiento, área en la que México es más vulnerable para la crítica internacional, dado que necesita mejorar la protección a los derechos laborales.

\footnotetext{
${ }^{17}$ Éste es el caso Gender, sometido a la NAO Estados Unidos en contra de México en 1997.
} 
Como he planteado anteriormente, una vez que se anunciaron las negociaciones del TLCAN, los activistas de Estados Unidos y sus contrapartes en México y Canadá se movilizaron y, finalmente, unieron sus fuerzas en un intento por tratar de influir en el curso del debate. La red de defensoría transnacional que a la postre se volvió activa en México no se interesó en este país a causa de los críticos mexicanos del tratado, sino más bien gracias a los grupos preocupados por los derechos humanos, laborales y por temas ambientales, entre otros, que tomaron ventaja de la oportunidad política que el tratado propiciaba para impulsar una agenda política (Stillerman, 2003).

Esto significa que la movilización transnacional en torno al tratado se dio en orden inverso (los activistas del norte se acercaron a sus contrapartes del sur), de tal modo que la represión, crucial en el modelo de derechos humanos, no se encuentra presente. Pero, una vez que hay defensores de los derechos humanos transnacionales en México, el modelo en espiral refleja adecuadamente el caso mexicano.

Como se menciona anteriormente, los Estados reaccionan, en primer lugar, ante la presión internacional negando la existencia de violaciones y rechazando la injerencia internacional en los asuntos locales. Sin embargo, al desarrollarse el caso de la red norteamericana contra el historial de derechos laborales en México, el gobierno de Salinas permaneció en silencio respecto a la aplicación de las normas sobre derechos laborales, aun cuando sí respondió a otras críticas por medio de reformas superficiales, como se prevé en la tercera fase. El rechazo de México a la intervención en asuntos laborales internos y sus llamados a la soberanía nacional aparecieron en la mesa de negociaciones, en donde este país casi renunció completamente a participar en el tratado a propósito de la recomendación de nuevas leyes laborales para la región de Norteamérica, y a causa de una propuesta de inspecciones trinacionales (Cameron y Tomlin, 2000).

La Стм, representante de México en los paneles de cláusulas sociales, se rehusó a participar en las mesas redondas para dar forma a los acuerdos (Mayer, 1998). Aún hoy en día, la oficina de la STPS que administra el ACLAN no participa en ninguna actividad no incluida específicamente en el anexo, porque considera que, al hacer visitas y audiencias públicas, Estados Unidos rebasa sus obligaciones en el ACLAN (NAO México, 2006).

Sin embargo, una vez que el NAALC fue implementado y que las redes de activistas transnacionales que fueron punta de lanza contra el TLCAN desarrollaron estrategias para llevar casos al panel, México fue obligado a ponerse a la defensiva para responder a las críticas en materia de prácticas de derechos laborales. Si bien este país hizo grandes esfuerzos para proyectar una imagen limpia durante las negociaciones del TLCAN e influir en la opinión pública que sobre él se tenía en Estados Unidos, considerando que casi todos los casos que se presentaron en primera instancia concernían a México, así como todas las denuncias sobre libertad de asociación, se percibió que 
su gobierno estaba tratando activamente de obstruir las tendencias democráticas del movimiento laboral mexicano en el proceso del ACLAN.

Las autoridades mexicanas a menudo tuvieron que responder a las acusaciones de violación a la libertad de asociación a través de canales presentados en los procesos del ACLAN. En la transición de la fase tres a la cuatro, los gobiernos dejan de negar que hubo violaciones a las normas y hacen frente a sus críticos para justificar las violaciones, luego de lo cual colaboran con ellos para mejorar la protección a las normas; al mismo tiempo, los agentes estatales comienzan a tomar en serio a los grupos internos como interlocutores, pues tienen detrás de sí el apoyo de importantes actores internacionales (Risse et al., 1999: 27-29). La dinámica "de abajo" que emerge en esta fase obliga a los Estados a implementar internamente las normas en la siguiente fase.

En México, la experiencia con el proceso del AClan cambió la dinámica política. La presentación de los casos confirió legitimidad a las agrupaciones pues, al ser escuchadas por personajes importantes del gobierno estadunidense, se convirtieron en actores de peso político dentro de México (Kay, 2005; Graubart, 2009). ${ }^{18}$ Como tal, el gobierno mexicano ya no pudo ignorar los esfuerzos de los grupos nacionales para obtener su atención y discutir estas cuestiones (Graubart, 2008; Hertel, 2006) sobre todo cuando, al revisar los casos, las NAO legitimaron tanto sus quejas como su participación en el proceso (Graubart, 2009).

Al finalizar la revisión de los casos de libertad de asociación interpuestos contra México, este país firmó cinco acuerdos ministeriales que indicaban su promesa de promover la protección de la libertad de asociación, incluyendo el acuerdo de 1997 de acceso a la información pública sobre documentos de registro. De acuerdo con Risse y sus coautores (1999), en la cuarta fase las acciones siguen a los discursos a fin de implementar normas internas. Al prometer dar seguimiento a las resoluciones de casos del proceso del ACLAN mediante acuerdos ministeriales, México estaría incluyendo entre las normas laborales la libertad de asociación, el uso de boletas secretas y el "estado prescriptivo" de la discriminación de género. Pero, al dar seguimiento a estas garantías escritas gracias a esfuerzos internos, garantizando las elecciones secretas, el fin por ley de las pruebas de embarazo y el recurso de discriminación de género mediante mecanismos de quejas, México ha asumido un compromiso que rebasa el estado prescriptivo. Es claro que las prácticas son desiguales, pues incluso estos ejemplos muestran que este país se encuentra detrás en materia de protección de la libertad de asociación, aunque el portal de registros es un paso en la dirección adecuada.

\footnotetext{
${ }^{18}$ Un importante ejemplo sucedió cuando la administración mexicana se rehusó a permitir que el equipo de la NAO pudiera entrevistar a las autoridades mexicanas durante una investigación del caso de Puebla en 2003. El equipo de la NAO tuvo que recurrir a archivos para encontrar la evidencia que estaban buscando (U.S. NAO, 2007).
} 
Aparentemente, estos casos dan idea de que, desde la entrada en vigor del TLCAN, México ha hecho algunos esfuerzos para demostrar su interés en el respeto a los derechos laborales; pero lo que sería decisivo es que México emprendiera reformas adicionales fuera de los mandatos y límites del ACLAN, si bien, por lo menos existe el indicio de que el país al menos había empezado a institucionalizar las normas de derechos laborales y sus prescripciones (aunque sólo en algunas áreas), por iniciativa propia.

Si México estuviera internalizando las normas de derechos laborales, sus efectos se harían notar como intentos de aplicar la normatividad en el área en la que ha sido más vulnerable a la crítica internacional y en la que más necesitaba mejorar su historial. De hecho, desde que comenzó el proceso del NAALC, el gobierno ha emprendido importantes avances en materia de aplicación de derechos laborales. Por ejemplo, ha aumentado continuamente la disponibilidad de recursos para inspecciones desde que se firmó el TLCAN.

Para consolidar estos esfuerzos, México empezó a incrementar su capacidad en cuanto a extender las prácticas para proteger los derechos laborales en todo el ámbito nacional. Aunque la información detallada sobre el presupuesto para inspección no está disponible antes de 2000, Franco Hijuelos (2001: 321) señala que, a partir de 1993 y hasta 1996, el presupuesto se incrementó en un 250 por ciento. La importancia de la inspección refleja un nuevo énfasis en el incremento entre 2000 y 2001, cuando los fondos asignados a la Dirección General de Inspección Federal aumentaron diez veces, y se mantuvo en ese nivel hasta 2009. ${ }^{19}$

Con el aumento del presupuesto, se produjo un aumento paralelo en la capacidad de inspección. Aunque hubo casi un tercio menos de inspectores después de la reorganización del departamento en 2000-2001, en ese último año se inspeccionaron 3023 lugares más de trabajo que en el 2000 (Secretaría del Trabajo y Previsión Social, 2007b). Lo que resulta interesante es que esta tendencia continuó durante la mayor parte de la década del 2000, pues cada año cada vez menos inspectores visitaban más lugares de trabajo. En el primer trimestre de 2009, habían sido inspeccionados 15432 lugares de trabajo, un aumento del 58 por ciento para el mismo periodo en 2008 (Secretaría del Trabajo y Previsión Social, 2007a). Mientras que la capacidad de inspección de México se considera todavía baja comparada con otros Estados en América Latina (Secretaría del Trabajo y Previsión Social, 2007b), estos datos otorgan evidencia para la afirmación de que el país está aumentando de manera decisiva su capacidad de inspección.

${ }^{19}$ Cálculo de la autora, a partir de los datos del presupuesto federal de México, 2000-2009. 
Como una segunda medida, es interesante anotar que se abrieron once juntas de conciliación y arbitraje en 1998, para fomentar un mayor acceso de los ciudadanos a los tribunales laborales (U.S. Department of State, 1999). Aunque, obviamente, el sistema de juntas es parte del problema de garantizar los derechos colectivos, la mayor parte de su responsabilidad reside en mediar las controversias sobre los derechos individuales entre trabajadores y administración, tales como disputas salariales y despidos ilegales. Por esta razón, el mayor acceso a las juntas laborales puede considerarse una medida positiva hacia la protección de los derechos laborales en México. Esta información apoya la idea de que desde que existe el TLCAN, México ha hecho esfuerzos para mostrar respeto por los derechos laborales al aumentar sus iniciativas internas para promover políticas y prácticas laborales que protegen estos derechos. Que estos esfuerzos hayan complementado las mejoras llevadas a cabo por el gobierno en el contexto del acuerdo NAALC, que incluso rebasen los compromisos que éste implica y que se hayan dado en el ámbito interior de manera independiente a las presiones transnacionales, también indica que los intentos de las autoridades mexicanas por mejorar su historial de derechos laborales son independientes de la presión del NAALC o de otras instancias internacionales.

\section{¿Ha Institucionalizado México las noRmas INTERnaCionales DE DERECHOS LABORALES?}

El uso del proceso del ACLAN en los tres países ha sido fundamental para constituir un foro donde se estudien cuestiones laborales, así como para mejorar los estándares laborales en México. Como describe el modelo, y como muestra el caso mexicano, las mejoras en algunas prácticas laborales fueron posibles, en parte, gracias al trabajo de los actores transnacionales, quienes presionaron a México por medio del ACLAN para realizar cambios al respecto. Al principio fueron superficiales, pero los consistentes esfuerzos durante el proceso del ACLAN empujaron al gobierno hacia reformas más profundas y, en algunos casos, a esfuerzos autónomos. Los elementos fundamentales del modelo -mayor escrutinio internacional y el trabajo de redes de defensa transnacionales- pueden ayudar a interpretar el aumento en la capacidad de respuesta de México con respecto a las violaciones laborales. Sin embargo, la discusión sobre la situación de los derechos laborales en este país durante los años del AcLAN analizados aquí sugiere que no ha internalizado por completo las normas internacionales de derechos laborales, o que su aceptación ha sido irregular, tanto en términos de las prácticas que han mejorado (esto es, las que han sido aceptadas con facilidad), como en los distintos niveles de gobernanza. 
¿Pueden estos patrones de resolución proveer evidencia de la socialización de normas en México? El modelo en espiral nos dice que estos cambios positivos pueden representar sólo intentos para desviar las críticas y, a su vez, indicar que la adopción de las normas de protección de los derechos laborales en México no ha avanzado de manera significativa. Sin embargo, para ciertos casos, la evidencia sugiere que la respuesta de su gobierno antes y después del ACLAN refleja la aceptación de las normas, y no es únicamente una respuesta instrumental a la crítica internacional.

Todo ello sugiere que México se está acercando a una mayor institucionalización de la protección de los derechos laborales, como se predice en las prescripciones de la etapa cuatro. Sin embargo, todavía hay muchos progresos por hacer y existen algunos retrocesos en el reconocimiento del derecho de libertad de asociación en los casos más recientes, lo cual evidencia que el gobierno no ha alcanzado aún la quinta etapa de institucionalización de las normas.

Por último, una de las principales críticas a los pactos sociales inscritos en acuerdos comerciales -como el ACLAN- es que éstos sólo son tan buenos como lo sean sus mecanismos de aplicación. Con frecuencia, los acuerdos carecen de "dientes" para castigar a los infractores o responder las quejas (Collingsworth, 2002). Mientras que la crítica se deriva de una posición normativa en la que los acuerdos laborales no son útiles si no se hacen cambios directos en el nivel de la empresa para recontratar trabajadores o realizar el pago de salarios caídos, estos aspectos de cumplimento no forman parte del acuerdo por razones de soberanía (Weiss, 2003). El análisis de los casos presentado aquí demuestra que la reparación directa era posible, aunque limitada. La experiencia del ACLAN ha dado lugar a cambios en las prácticas laborales en México, lo que podría traducirse en una mayor protección para los trabajadores mexicanos a largo plazo.

En efecto, el acuerdo paralelo creó oportunidades para que las redes transnacionales de defensa establecieran en México un camino para mejorar la aplicación de los derechos laborales, independientemente de qué tan fuerte o efectivo era el acuerdo per se, en términos de sancionar un cumplimiento ligero. Es debido a estos efectos secundarios generados por la presión internacional que los defensores pueden seguir buscando la protección de los derechos laborales mediante los acuerdos comerciales como un camino adicional para mejorar el cumplimiento de dichos derechos. 


\section{Biblografía}

ASPINWALL, MARK

2013 Side Effects: Mexican Governance under NAFTA's Labor and Environmental Agreements, Palo Alto, Calif., Stanford University Press.

BABSON, STEVE

2002 "Free Trade and Worker Solidarity in the North American Auto Industry", en Bruce Nissen, ed., Unions in a Globalized Environment: Changing Borders, Organizational Boundaries, and Social Roles, Londres, M.E. Sharpe, pp. 17-44.

Baer, M. Delal y Sidney Weintraub

1994 "The Pressures for Political Reform in Mexico" en M. Delal Baer y Sidney Weintraub, eds., The NAFTA Debate: Grappling with Unconventional Trade Issues, Boulder, Colo., Lynne Rienner, pp. 159-180.

\section{BORDERLINES}

1997 "Maxi-Switch Workers Win Union Recognition", en <http://www.americaspolicy.org/borderlines/1997/bl37/bl7bbbody.html>, consultada el 13 de febrero de 2000 y disponible con la autora.

BRYSK, ALISON

1993 "From Above and Below: Social Movements, The International System and Human Rights in Argentina", Comparative Political Studies, vol. 26, no. 3, pp. 259-285.

Cameron, Maxwell A. y Brian W. Tomlin

2000 The Making of NAFTA: How the Deal Was Done, Ithaca, Cornell University Press.

CAMP KeITH, LindA

1999 "The United Nations International Covenant on Civil and Political Rights: Does it Make a Difference in Human Rights Behavior?", Journal of Peace Research, vol. 36, no.1, pp. 95-118.

Clark, Ann Marie, Elisabeth Friedman y Kathryn Hochstetler

1998 "The Sovereign Limits of Global Civil Society: A Comparison of NGO Participation in UN World Conferences on the Environment, Human Rights and Women", en World Politics, vol. 51, no. 1, pp. 1-35. 
COLLINGSWORTH, TERRY

2002 "The Key Human Rights Challenge: Developing Enforcement Mechanisms", Harvard Human Rights Journal, vol. 15, pp. 183-210, primavera.

COMPa, LANCE

2001 "NAFTA's Side Labor Agreement and International Labor Solidarity", Antipode, vol. 33, no. 3, pp. 451-467.

Covarrubias Velasco, Ana

1999 "El problema de los derechos humanos y los cambios en la política exterior", Foro Internacional, vol. 39, no. 4, pp. 429-452.

COOK, MARIA LORENA

1997 "Regional Integration and Transnational Politics: Popular Sector Strategies in the NAFTA Era", en Vilas, Hite, Martin et al., coords., The New Politics of Inequality in Latin America, Nueva York, Oxford University Press, pp. 516-541.

Domínguez, Jorge I. y Rafael Fernández de Castro

2009 The United States and Mexico: Between Partnership and Conflict, Nueva York, Routledge.

FINNEMORE, MARTHA

1996 National Interests in International Society, Ithaca, Cornell University Press.

GraubarT, JONATHAN

2009 “The Legalization of Transnational Political Opportunity Structures: Mobilization of NAFTA's Labor Citizen Petitions for Domestic Political Gain”, en Laura Mac Donald y Jeffrey Ayres, eds., Contentious Politics in North America, Nueva York, Palgrave, pp. 177-194.

2008 Legalizing Transnational Activism: The Struggle to Gain Social Change from NAFTA's Citizen Petitions, University Park, Penn., University of Pennsylvania Press.

Gurowitz, AMY

1999 "Mobilizing International Norms: Domestic Actors, Immigrants, and the Japanese State", World Politics, vol. 51, no. 3, pp. 413-445. 
HaAs, Peter

1989 “Do Regimes Matter? Epistemic Communities and Mediterranean Pollution Control", en International Organization, vol. 43, no. 3, pp. 377-403.

Hafner-Burton, EMilie M.

2009 Forced to be Good: Why Trade Agreements Boost Human Rights, Ithaca, Cornell University Press.

2008 "Sticks and Stones: Naming and Shaming the Human Rights Enforcement Problem", International Organization, vol. 62, no. 4, pp. 689-716.

2005 "Trading Human Rights: How Preferential Trade Agreements Influence Government Repression", International Organization, vol. 59, no. 3, pp. 593-629.

Hathaway, Dale

2002 "Mexico's Frente Autentico del Trabajo and the Problem of Unionizing Maquiladoras", en Labor History, vol. 43, no. 4, pp. 427-438.

HATHAWAY, OONA

2002 "Do Human Rights Treaties Make a Difference?", The Yale Law Journal, vol. 111, no. 3, pp. 1935-2042.

HeRTEL, SHAREEN

2006 Unexpected Power: Conflict and Change among Transnational Activists, Ithaca, Cornell University Press.

2003 "Una contienda acotada: la defensa transnacional de los derechos laborales de las mujeres en las maquiladoras de México", Región y Sociedad, vol. 26, no. 16, pp. 153-191.

HILL, DANNY

2009 "Estimating the Effect of Human Rights Treaties on State Behavior", ponencia presentada en la $67^{\mathrm{a}}$ Conferencia Nacional de la Midwest Political Science Association, Chicago, Ill, 2-4 de abril.

Jepperson, Ronald, Alexander Wendt y Peter Katzenstein

1996 "Norms, Identity, and Culture in National Security" en Ronald Jepperson, Alexander Wendt y Peter Katzenstein, eds., The Culture of National Security, Nueva York, Columbia University Press, pp. 33-75. 
JOPPKE, CHRISTIAN

1998 "Why Liberal States Accept Unwanted Immigration", World Politics, vol. 50, no. 2, pp. 266-293.

JuÁREZ NúÑEZ, HubERTO

2002 "Maquila Workers in Mexico: The Prospects of Organization and International Solidarity", Labor History, vol. 43, no. 4, pp. 439-450.

KAY, TAMARA

2011 NAFTA and the Politics of Labor Transnationalism, Nueva York, Cambridge University Press.

2005 "Labor Transnationalism and Global Governance: The Impact of NAFTA on Transnational Labor Relationships in North America", American Journal of Sociology, vol. 111, no. 3, pp. 715-756.

Keck, Margaret y Kathryn SikKinK

1998 Activists Beyond Borders: Advocacy Networks in International Politics, Ithaca, Cornell University Press.

Kowert, Paul y JefFrey Legro

1996 "Norms, Identity and Their Limits: A Theoretical Reprise," en Peter J. Katzenstein, ed., The Culture of National Security, Nueva York, Columbia University Press, pp. 451-497.

MAYer, Frederick

1998 Interpreting nafta: The Science and Art of Political Analysis, Nueva York, Columbia University Press.

Murillo, Victoria M.

2001 Labor Unions, Partisan Coalitions, and Market Reforms in Latin America, Nueva York, Cambridge University Press.

National Administrative Office (naO), México

2006 Entrevista, Ciudad de México, 16 de julio.

National Administrative Office (naO), United States

2007 Entrevista, Washington, D.C., $1^{\circ}$ de julio. 
North American Agreement on Labor CoOperation (naAlC)

1993 North American Agreement on Labor Cooperation (NAALC): Between the Government of the United States of America, the Government of Canada, and the Government of the United Mexican States.

Nolan García, Kimberly A.

2011 “The Evolution of United States-Mexico Labor Cooperation (1994-2009): Achievements and Challenges", Politics and Policy, vol. 39, no. 1, pp. 91-117.

PRICE, RICHARD

1998 "Reversing the Gun Sights: Transnational Civil Society Targets Land Mines", International Organization, vol. 52, no. 3, pp. 613-644.

Price, Richard y Nina TANnenwald

1996 "Norms and Deterrence: The Nuclear and Chemical Weapons Taboos", en Peter J. Katzenstein, ed., The Culture of National Security: Norms and Identity in World Politics, Nueva York, Columbia University Press, pp. 114-152.

Risse, Thomas, Steven Ropp y Kathryn Sikkink

1999 The Power of Human Rights: International Norms and Domestic Change, Nueva York, Cambridge University Press.

Roman, Richard y Edur Velasco Arregui

2006 "The State, the Bourgeoisie, and the Unions: The Recycling of Mexico's System of Labor Control", Latin American Perspectives, vol. 33, no. 2, pp. 95-103.

Secretaría del Trabajo y Previsión Social

s.f. a <http:// registrodeasociaciones.stps.gob.mx/regaso/ consultaregasociaciones.asp $>$, consultada el 10 mayo de 2013.

s.f.b <http:// contratoscolectivos.stps.gob.mx/RegAso/legal_contratos.asp>, consultada el 10 de mayo de 2013.

2007a Avances, stPs México, Ciudad de México.

2007b "Solicitante identificado con folio en el SISI 001400083407", Oficio no. 0001400083407, disponible con la autora.

SHIRK, David A. y Emily Edmonds-Poli

2009 Contemporary Mexican Politics, Lanham, Maryland, Rowman and Littlefield. 
SHOR, ERAN

2008 "Conflict, Terrorism, and the Socialization of Human Rights Norms: The Spiral Model Revisited", Social Problems, vol. 55, no.1, pp. 117-138.

STILLERMAN, JOEL

2003 "Transnational Activist Networks and the Emergence of Labor Internationalism in the NAFTA Countries", en Social Science History, vol. 27, no. 4, pp. 577-601.

U.S. Department of Labor, International Labor AfFairs Bureau

2007 Public Report of Review of NAO Submission 2005-03 (Hidalgo), Washington, D.C., US NAO.

U.S. Department of State, Bureau of Democracy, Human Rights and Labor

2000 "Country Reports: Mexico", en <http:/ / www.state.gov/g/drl/rls/hrrpt/2002/ 18338pf.htm>, consultada el 21 de enero de 2001.

1999 “Country Reports: Mexico", en <http:/ / www.state.gov/g/drl/rls/hrrpt/2002/ 18338pf.htm>, consultada el 21 de enero de 2001.

WeIsS, MARLEY S.

2003 “Two Steps Forward, One Step Back or Vice Versa: Labor Rights under Free Trade Agreements from NAFTA through Jordan via Chile to Latin America and Beyond", University of San Francisco Law Review, vol. 37, pp. 689-755.

WiLliams, HeATHER L.

2003 "Labor Tragedy and Legal Farce: The Han Young Struggle in Tijuana, Mexico", Social Science History, vol. 27, no. 4, pp. 525-550.

1999 "Mobile Capital and Transborder Labor Rights Mobilization", Politics and Society, vol. 27, no. 1, pp. 39-66.

ZAPATA, FRANCISCO

2006 “La negociación de las reformas a la Ley Federal del Trabajo (1989-2005)”, Foro Internacional, vol. 46, no. 1, pp. 81-102. 